\title{
A Simplified Method for (Circular) Transmission Line Model Simulation and Ultra-low Contact Resistivity Extraction
}

\author{
Hao Yu, Marc Schaekers, Tom Schram, Nadine Collaert, Kristin De Meyer, Fellow, IEEE, Naoto \\ Horiguchi, Aaron Thean and Kathy Barla
}

\begin{abstract}
The metal resistance in the transmission line model (TLM) structures creates a serious obstacle to determine precisely the intrinsic contact resistivity. To tackle this problem, we propose a new model, the Lump Model, to evaluate the metal resistance influence in both TLM and circular TLM (CTLM) test structures. In this work, we demonstrate the high simplicity, great robustness and flexibility of the Lump Model. The previous reported contact resistivity values extracted with CTLM are usually above $1 \times 10^{-7} \Omega \cdot \mathrm{cm}^{2}$ because the metal resistance impact is commonly neglected. This is the first time that the role of the metal in CTLM is appropriately analyzed. Low contact resistivity, $3.6 \times 10^{-8} \Omega \cdot \mathrm{cm}^{2}$, of standard NiSi/n-Si contact has been extracted and this shows the high sensitivity of this method.
\end{abstract}

Index Terms-Contact resistance, ohmic contact, transmission line model, circular transmission line model, simulation

\section{INTRODUCTION}

$\mathrm{T}$ he International Technology Roadmap for Semiconductors (ITRS) has put forward a requirement of contact resistivity below $1 \times 10^{-8} \Omega \cdot \mathrm{cm}^{2}$ for the $10 \mathrm{~nm}$ technology node and beyond [1]. Besides this challenge, the ultra-low contact resistivity has also increased the requirement of precise measurement.

The transmission line model (TLM) [2] and the circular transmission line model (CTLM) [3] are the two most commonly applied methods to extract the contact resistivity, $\rho_{c}$, from the contact resistance, $R_{c}$. For higher values of $R_{c}$, the metal resistance impact is negligible. However, when $R_{c}$ becomes lower, neglecting the metal resistance can easily lead to a misinterpretation of $R_{c}$ and an inaccurate extraction of $\rho_{c}$. Marlow et al. have developed an analytical model to describe the role of the metal resistance in TLM [4]. Further, Dormaier et al. proposed a refined TLM structure to minimize the metal resistance influence [5]. Compared with TLM, CTLM benefits from its easier process and especially suits the quick contact scheme screening. However, the metal resistance influence in CTLM has never been properly analyzed.

Manuscript submitted April 6, 2014. This work was supported by imec's Core program on LOGIC DEVICES.

H. Yu and K. De Meyer are with imec, 3001 Leuven, Belgium,and also with the Department of Electrical Engineering, Katholieke Universiteit (K.U.), 3001 Leuven, Belgium (e-mail: hao.yu@imec.be).

M. Schaekers, T. Schram, N. Collaert, N. Horiguchi, A. Thean and K. Barla are with imec, 3001 Leuven, Belgium.
In this work, a simplified model, the Lump Model (LM), is designed to facilitate the simulation of the TLM and CTLM test structures. Assisted by the LM simulation, the low $\rho_{c}$ of standard $\mathrm{NiSi} / \mathrm{n}-\mathrm{Si}$ contact, $3.6 \times 10^{-8} \Omega \cdot \mathrm{cm}^{2}$, is successfully extracted with CTLM. Previously, the contact resistivity values extracted with CTLM were usually above $1 \times 10^{-7}$ or $1 \times 10^{-6}$ $\Omega \cdot \mathrm{cm}^{2}[6,7]$. To our knowledge, this is the first time that the ultra-low $\rho_{c}$ of metal-semiconductor (MS) contacts is properly determined by the CTLM method. The sensitivity of the CTLM method is therefore improved to be comparable with other state-of-the-art contact resistivity extraction models. [5]

\section{EXPERIMENTS}

$300 \mathrm{~mm}$ blanket p-type silicon wafers received an ion implantation with phosphorus $\left(1.8 \mathrm{keV}, 1 \times 10^{13} \mathrm{~cm}^{-2}\right)$ and arsenic $\left(20 \mathrm{keV}, 3 \times 10^{15} \mathrm{~cm}^{-2}\right)$. A post high temperature activation annealing at $1035^{\circ} \mathrm{C}$ was performed in He. CTLM patterns were defined by lithography and etching with a dielectric hardmask. A $6 \mathrm{~nm}$ thin $\mathrm{Ni}(\mathrm{Pt})$ layer was deposited by sputtering, followed by a two-step silicidation process - a first 1 min $275{ }^{\circ} \mathrm{C}$ annealing in $\mathrm{N}_{2}$ ambient was followed by a wet etching removal of $\mathrm{Ni}$ residue and a second $1 \mathrm{~min} 400{ }^{\circ} \mathrm{C}$ annealing in $\mathrm{N}_{2}$ ambient. The standard liner stack was then deposited. Following the stack deposition, a thick layer of $\mathrm{Cu}$ or W was deposited by plating or by chemical vapor deposition (CVD). Chemical-mechanical planarization (CMP) was then performed until $\sim 150 \mathrm{~nm}$ thick metal remained. An $8 \mathrm{~nm} \mathrm{SiC}$ layer was deposited on $\mathrm{Cu}$ to prevent the oxidation. All electrical measurements were performed using an HP4156c.

\section{METHODOLOGY}

The top and sectional schematics of the CTLM test structure are shown in Fig. 1a. The total resistance $\left(R_{T}\right)$ of CTLM is defined as the resistance between the two probes, $\mathrm{P}_{1}$ and $\mathrm{P}_{2}$. In reality, a four-point-probe (4pp) measurement is applied to eliminate the probe series resistance impact. $R_{T}$ is measured with various spacing $(s)$ between the metal electrodes. Two CTLM $R_{T}-s$ curves of the same NiSi/n-Si contacts with the different cap metal were compared in Fig. 1b. The metal sheet resistance, $R_{m}$, on the $\mathrm{W}$ or Cu capped samples is $\sim 1.5$ and $\sim 0.2$ $\Omega /$ sq, respectively. A difference in $R_{T}-s$ relation caused by the cap metal can be observed. Previous reports [6,7] commonly assume that the $R_{T}-s$ relation of CTLM structures is determined only by two factors - the substrate sheet resistance $\left(R_{s}\right)$ and $\rho_{c}$. 
However, the impact of $R_{m}$ should also be included, otherwise the large deviation of the $R_{T}-s$ relation caused by $R_{m}$ will lead to a serious inaccuracy in the extraction of $\rho_{c}$.

Marlow et al. have developed an analytical model including the $R_{m}$ influence for TLM. [4] However, because of the special pattern of CTLM test structures, there has been no analytical or numeric method to describe the role of $R_{m}$ in CTLM. Alternatively, we use the TCAD simulations for the investigation of the CTLM structure. There are two major challenges for the CTLM simulation; (1) a large amount of nodes are needed for the intensive current density and complex electric field distribution and (2) the large dimension and the circular pattern. To overcome these challenges, a simplified substitute model, the Lump Model (LM), is developed to realize the simulation of both the TLM and the CTLM behavior. First, the feasibility and validity of 1D LM is checked with TLM. Then 2D LM is applied for CTLM simulation.

LM is a simple model consisting of several lumps of resistors in series to simulate the complex behavior of TLM and CTLM. The dimension and the resistance of the lumps are the two major parameters to be defined. To mimic the behavior of the TLM, LM is simply a $1 \mathrm{D}$ model. The construction of the LM is based on the current crowding effect at the contact edge, as illustrated by Fig. 2 . The current distribution at the contact edge is derived based on the model given by Marlow et al. [4]

$I(x)=C_{1}+C_{2} \exp [(d-x) / a]+C_{3} \exp [-(d-x) / a]$ where $\mathrm{x}$ is the distance from the edge, $d$ is the length of the metal electrode,

$$
\begin{gathered}
a=\sqrt{\rho_{c} /\left(R_{s}+R_{m}\right)}, \\
C_{1}=R_{m} I(0) /\left(R_{s}+R_{m}\right), \\
C_{2}=C_{1}\left(R_{s} / R_{m}\right) \exp (-d / a), \\
C_{3} \approx-C_{1}(\text { when } d / 4 \gg a) .
\end{gathered}
$$

As illustrated by Fig. 2, when $\rho_{c}$ is low, all the current crowds into the metal within a small area at the contact edge. We notice that, due to the current crowding effect, the distance that every piece of current flows within the substrate is almost the same, compared to the complete TLM dimension. As a result, we make an appropriate assumption that at the edge of the contact all pieces of the current travel the same distance, $\bar{L}$, along the substrate and then flow into the metal together. $\bar{L}$ can be calculated from (1)

$$
\bar{L}=\int x d i / I(0)=R_{s} L_{t} /\left(R_{s}+R_{m}\right) \approx L_{t}\left(R_{m} \ll R_{s}\right)
$$
where $L_{t}$ is the well-known transfer length of the TLM structure

$$
L_{t}=\sqrt{\rho_{c} / R_{s}} .
$$

The complete TLM structure can thus be simplified into three lumps in series - two metal lumps with the sheet resistance of $R_{m}$ and one semiconductor lump in between with the sheet resistance $R_{s}$. The lengths of the metal lump and the semiconductor lump are $d-L_{t}$ and $s+2 L_{t}$, respectively. In LM, the role of $\rho_{c}$ is represented by $L_{t}$, as shown by (3). As a result, LM is essentially a simulation model including all three factors of $\rho_{c}, R_{s}$ and $R_{m}$. The inset of Fig.2 schematically shows the TLM and the LM structures.

To verify the robustness of both the LM and the complete TLM structure simulations, the $R_{T}-s$ relations are examined and compared with the numerical model developed by Marlow et al. [4]. At the same time, the role of $R_{m}$ in the TLM structure is studied. We will look at the example of $\rho_{c}=1 \times 10^{-8} \Omega \cdot \mathrm{cm}^{2}$ and $R_{s}=205 \Omega /$ sq using three $R_{m}$ values $-0,0.2$, and $2.0 \Omega / \mathrm{sq}$. A $100 \mu \mathrm{m}$ electrode length and a set of spacing values are applied. As shown in Fig. 3, a good agreement is achieved between simulation and calculation. In practice, the LM simulation dramatically reduces the computation time needed as compared to the complete TLM simulation. As for the role of metal resistance, $R_{m}$ causes an upwards shift of the $R_{T}-s$ curve. A high $R_{m}$ can even dominate the intercept of the curve.

After the verification of using a 1D LM to simulate the TLM behavior, it is clear that we can distinguish the metal and the semiconductor contribution with independent lumps. This simplification dramatically reduces the simulation complexity. As for CTLM, the simulation of a complete structure is much more difficult to realize. The major challenge is to analyze the current and field distribution in the large 3D pattern. Fortunately, we notice that, similarly with TLM, the transfer of current between the metal and the semiconductor in CTLM only happens at the contact edge and we can thus also separate the metal and semiconductor parts with a 2D LM. The settings of the 2D LM parameters are shown in Fig. 4a. The parameter $L_{t l}$ and $L_{t 2}$ are the transfer length inside and outside the ring, respectively. Due to the fact that the radius of the circular electrode $(r)$ is far larger than the transfer length, $L_{t 1}$ and $L_{t 2}$ are almost the same and can be both derived by (3). [4] Fig. 4b shows the $R_{T}-S$ curves derived by 2D LM simulation. $R_{s}=205$ $\Omega / \mathrm{sq}$ and $\rho_{c}=1 \times 10^{-8} \Omega \cdot \mathrm{cm}^{2}$ are applied in simulation. In the ideal case, $R_{m}=0$ and the inner and outer metal electrodes are equipotential. The voltage drops totally over the semiconductor channel and contacts of CTLM. But in reality, the resistance of the metal electrode will also cause a voltage drop. Fig. $4 \mathrm{c}$ and Fig. $4 \mathrm{~d}$ show the electric potential contour plots when $R_{m}=0.2$ $\Omega / \mathrm{sq}$ and $R_{m}=2 \Omega / \mathrm{sq}$. As shown in Fig. $4 \mathrm{~d}$, when $R_{m}$ is high, the large voltage drop over the metal can suppress the effective voltage drop on the ring. Like in the TLM case in Fig. 3, $R_{m}$ causes an upward shift of the CTLM $R_{T}-s$ curves in Fig. $4 \mathrm{~b}$,

In previous contact studies [6,7], for both TLM and CTLM, $R_{T}-s$ curves are usually interpreted with only $R_{s}$ and $\rho_{c}$. From the intercept, $\rho_{c}$ is extracted. However, as shown by Fig. 3 and Fig. $2 \mathrm{~b}$, neglecting the metal influence will eventually lead to a serious inaccuracy of the $\rho_{c}$ extraction, especially with high $R_{m}$ (>1 $\Omega / \mathrm{sq}$ ). With simulation, the role of $R_{m}$ in both TLM and CTLM can be analyzed and the $\rho_{c}$ extraction can be refined. Nevertheless, $R_{m}$ still serves as a parasitic factor and should be minimized to enhance the sensitivity of the $\rho_{c}$ test structure. Therefore, we have switched the cap metal from $\mathrm{W}(\sim 1.5 \Omega / \mathrm{sq})$ to $\mathrm{Cu}(\sim 0.16 \Omega / \mathrm{sq})$.

Assisted by the 2D LM simulation, the CTLM $R_{T}-s$ curves can successfully be derived with values of $\rho_{c}, R_{s}$ and $R_{m}$. A new $\rho_{c}$ extraction method can be developed based on electrical measurements and simulations. In the practical experiments, $R_{m}$ and $R_{T}-S$ data sets can be derived using the $4 \mathrm{pp}$ measurement. Then by modulating the $R_{s}$ and $\rho_{c}$ parameter values in the $2 \mathrm{D}$ $\mathrm{LM}$, the trend of the measured $R_{T}-s$ data sets can be simulated and the intrinsic $R_{s}$ and $\rho_{c}$ values can be determined. To examine this method, we applied it to the standard low- $\rho_{c}$ $\mathrm{NiSi} / \mathrm{nSi}$ contact with $150 \mathrm{~nm} \mathrm{Cu}$ cap. $R_{m}$ and six sets of $R_{T}-s$ 
values were measured. In this case, $R_{m}$ is $0.16 \Omega / \mathrm{sq}$. Both the measurement results and the simulation curves are shown in Fig.5. For both dimensions of the CTLM, $\rho_{c}=3.6 \times 10^{-8} \Omega \cdot \mathrm{cm}^{2}$ and $R_{s}=118.6 \Omega / \mathrm{sq}$ are determined, which shows high sensitivity of our $\rho_{c}$ extraction method. For silicide contacts, two additional factors caused by silicidation are neglected in the $\rho_{c}$ extraction procedures above- the lateral penetration of $\mathrm{NiSi}$ and the dopant segregation. The influence of both factors is small since the $\mathrm{Ni}(\mathrm{Pt}) \mathrm{Si}$ layer in our experiment is as thin as $\sim 11 \mathrm{~nm}$. Accounting for the mixed effects of these two factors, small changes can be made in the 2D LM simulation and there would be a correction of approximately $\pm 0.4 \times 10^{-8} \Omega \cdot \mathrm{cm}^{2}$ from the $\rho_{c}$ results above.

\section{CONCLUSION}

The simple and robust Lump Model was presented to facilitate the simulation of both TLM and CTLM. Based on the Lump Model simulation, the total resistance-spacing $\left(R_{T}-S\right)$ relations of CTLM were derived successfully including the influence of the metal. From the simulations it is clear that the metal sheet resistance needs to be reduced as much as possible thereby favoring metals like $\mathrm{Cu}$ and $\mathrm{Al}$. Furthermore, we have developed a $\rho_{c}$ extraction method combined with the Lump Model simulation, which can be used to determine ultra-low contact resistivities from the CTLM structures. The method has been demonstrated for a NiSi/n-Si contact with a $\rho_{c}$ of $3.6 \times 10^{-8}$ $\Omega \cdot \mathrm{cm}^{2}$. To our knowledge, this is the lowest $\rho_{c}$ that has been reliably extracted using CTLM structures. This is also the first time that the $R_{m}$ impact in CTLM is successfully analyzed.

[1] The International Technology Roadmap for Semiconductors (ITRS), 2013 [2] G. K. Reeves, "Specific contact resistance using a circular transmission line model", Solid-State Electron., vol. 23, pp. 487-490, 1980

[3] G. K. Reeves and H. B. Harrison, "Obtaining the specific contact resistance from transmission line model measurements", IEEE Electron Device Lett., vol. EDL-3, no. 5, May 1982

[4] G. S. Marlow and M. B. Das, "The effets of contact size and non-zero metal resistance on the determination of specific contact resistance", Solid-State Electron., vol. 25, no. 2, pp. 91-94, 1982

[5] R. Dormaier and S. E. Mohney, "Factors controlling the resistance of ohmic contacts to n-InGaAs", J. Vac. Sci. Technol. B, vol. 30, no. 3, pp. 031209-1-031209-10, May/Jun 2012

[6] A. Firrincieli, K. Martens, R. Rooyackers, et al., "Study of ohmic contacts to n-type Ge: snowplow and laser activation", Appl. Phys. Lett., vol. 99, pp. 242104-1-142104-3, Dec. 2011

[7] F. Liu, B. Hsia, C. Carraro, et al., "Enhanced ohmic contact via graphitization of polycrystalline silicon carbide", Appl. Phys. Lett., vol. 97, pp. 262107-1-262107-3, Dec. 2010
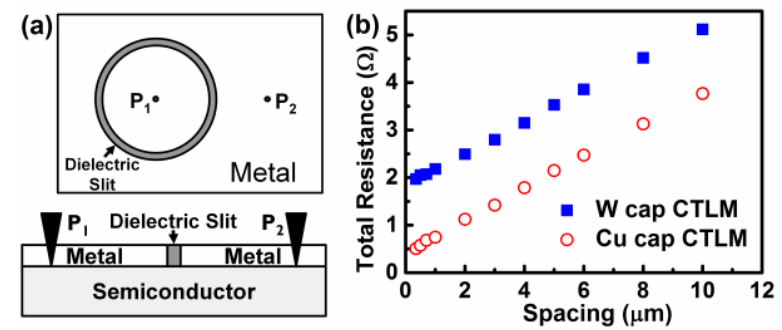

Fig. 1. (a) The schematic top view and sectional view of the CTLM test pattern. $\mathrm{P}_{1}$ and $\mathrm{P}_{2}$ are the probes of the current source. (b) CTLM $R_{T}-s$ curves of $\mathrm{NiSi} / \mathrm{n}-\mathrm{Si}$ contacts with $\mathrm{Cu}$ and $\mathrm{W}$ cap layers. The spacing is the distance between metal electrodes.

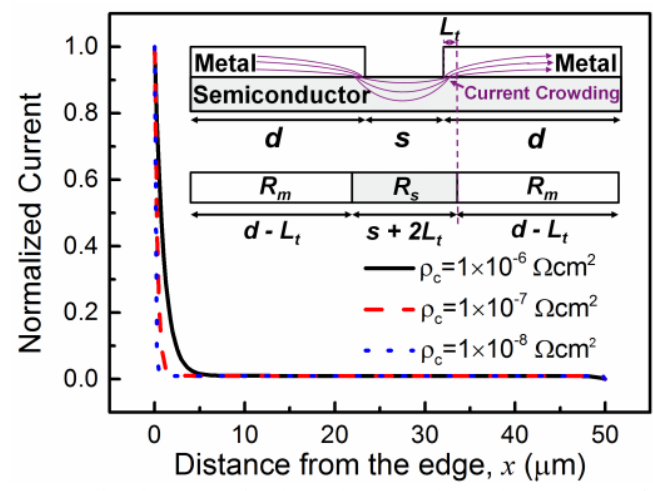

Fig.2. The normalized current in the substrate along the contact of TLM. The insets are the schematics of the complete TLM structure and LM.

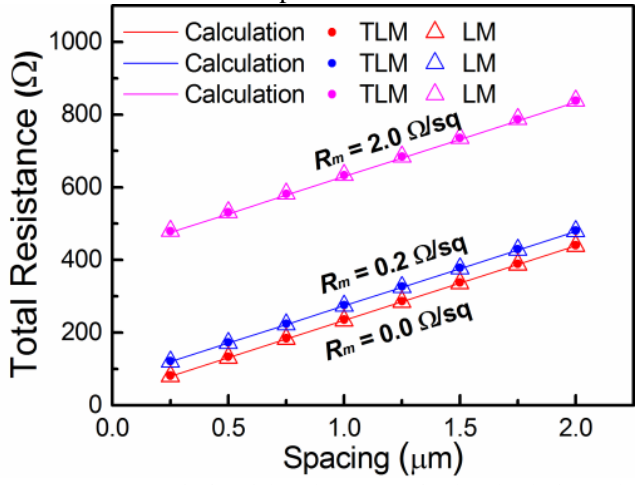

Fig. 3. The $R_{T}-s$ curves derived by the numerical calculation, the complete TLM simulation and the LM simulation.
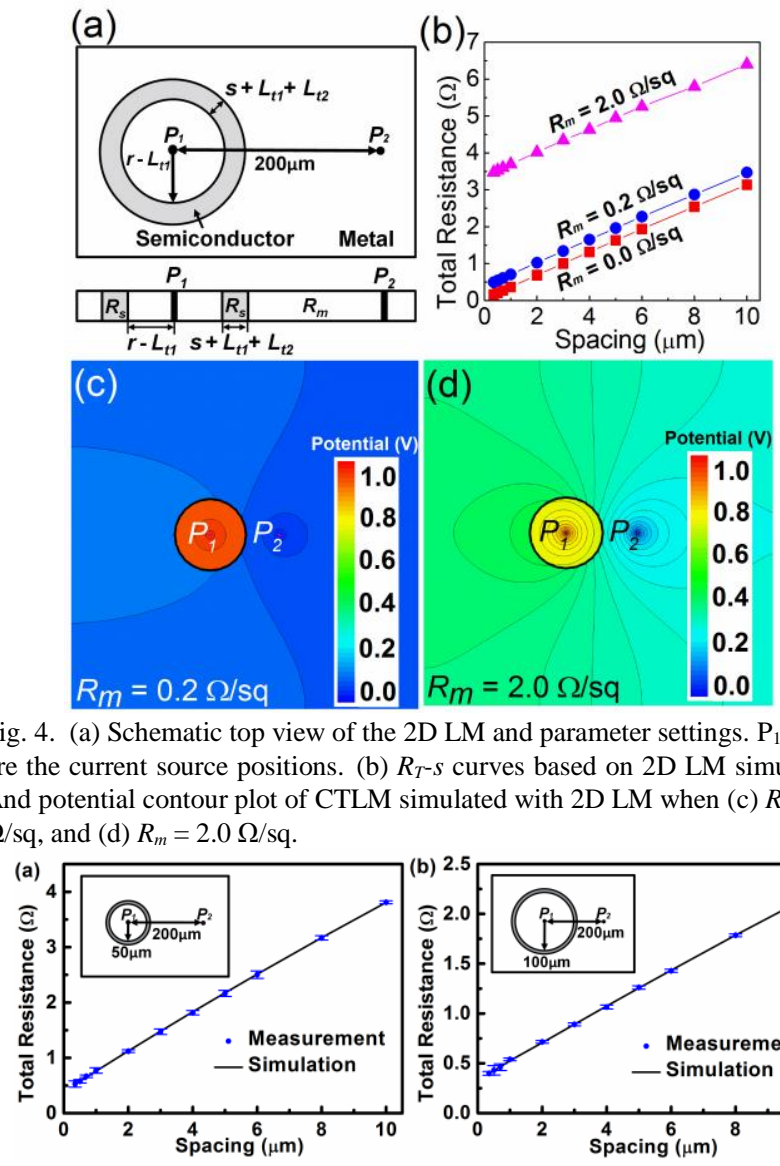

Fig. 4. (a) Schematic top view of the $2 \mathrm{D} L M$ and parameter settings. $\mathrm{P}_{1}$ and $\mathrm{P}_{2}$ are the current source positions. (b) $R_{T}-s$ curves based on 2D LM simulation. And potential contour plot of CTLM simulated with 2D LM when (c) $R_{m}=0.2$ $\Omega / \mathrm{sq}$, and (d) $R_{m}=2.0 \Omega / \mathrm{sq}$.

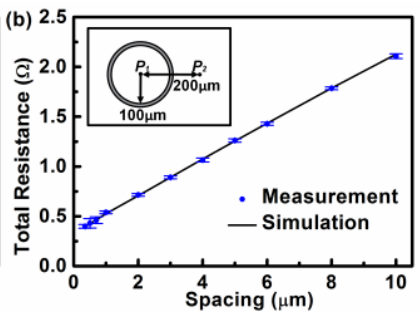

Fig. 5. The measurement and the 2D LM simulation results for CTLM of two dimensions - the circle inner radius is (a) $50 \mu \mathrm{m}$ and (b) $100 \mu \mathrm{m}$, respectively. The short error bars exhibit the high repeatability of the measurements. The insets are the schematic top views of the CTLM structures. 